\title{
A positive proof of the Littlewood-Richardson rule using the octahedron recurrence
}

\author{
Allen Knutson \\ Mathematics Department, UC Berkeley, Berkeley, California \\ allenk@math . berkeley .edu \\ Terence Tao \\ Mathematics Department, UCLA, Los Angeles, California \\ tao@math.ucla.edu \\ Christopher Woodward \\ Mathematics Department, Rutgers University, New Brunswick, New Jersey \\ ctw@math.rutgers.edu \\ * \\ Submitted: Jun 18 2003; Accepted: Jul 4, 2004; Published: Sep 13, 2004 \\ Mathematics Subject Classifications: 52B20, 05C05
}

\begin{abstract}
We define the hive ring, which has a basis indexed by dominant weights for $\mathrm{GL}_{n}(\mathbb{C})$, and structure constants given by counting hives [Knutson-Tao, "The honeycomb model of $\mathrm{GL}_{n}$ tensor products"] (or equivalently honeycombs, or BZ patterns [Berenstein-Zelevinsky, "Involutions on Gel'fand-Tsetlin schemes..."]).

We use the octahedron rule from [Robbins-Rumsey, "Determinants..."] to prove bijectively that this "ring" is indeed associative.

This, and the Pieri rule, give a self-contained proof that the hive ring is isomorphic as a ring-with-basis to the representation ring of $\mathrm{GL}_{\mathfrak{n}}(\mathbb{C})$.

In the honeycomb interpretation, the octahedron rule becomes "scattering" of the honeycombs. This recovers some of the "crosses and wrenches" diagrams from Speyer's very recent preprint ["Perfect matchings..."], whose results we use to give a closed form for the associativity bijection.
\end{abstract}

*AK was supported by NSF grant 0072667, and a Sloan Fellowship. TT was supported by the Clay Mathematics Institute, and the Packard Foundation. CW was supported by NSF grant 9971357. 


\section{Contents}

1 Introduction $\quad 2$

1.1 Acknowledgements. . . . . . . . . . . . . . . . 3

2 Hives $\quad 3$

3 Recognizing the representation ring $\operatorname{Rep}\left(\mathrm{GL}_{n}(\mathbb{C})\right) \quad 5$

4 The hive ring satisfies the $\operatorname{det}^{-1}$ and Pieri rules $\quad 6$

5 The hive ring is associative $r$

6 The honeycomb interpretation: scattering $\quad 11$

6.1 Honeycomb scattering vs. hive excavation. . . . . . . . . . . . . . . . 14

6.2 The scattering rule in $[\mathrm{GP}] . \ldots \ldots \ldots \ldots \ldots$

$\begin{array}{lll}7 & \text { A closed form for the associativity bijection } & 14\end{array}$

\section{Introduction}

Let $\operatorname{Rep}\left(\mathrm{GL}_{n}(\mathbb{C})\right)$ denote the ring of (formal differences of algebraic finite-dimensional) representations of $\mathrm{GL}_{n}(\mathbb{C})$, with addition and multiplication coming from direct sum and tensor product of representations. Then $\operatorname{Rep}\left(\mathrm{GL}_{\mathfrak{n}}(\mathbb{C})\right)$ has a canonical basis $\left\{\left[\mathrm{V}_{\lambda}\right]\right\}$, the irreducible representations, indexed by the set $\mathbb{Z}_{\text {dec }}^{n}$ of weakly decreasing $\boldsymbol{n}$-tuples of integers. (The "[]" are only there to maintain a proper distinction between an actual representation $V_{\lambda}$ and the corresponding element of $\operatorname{Rep}\left(G L_{n}(\mathbb{C})\right.$ ), which is really an isomorphism class.) Our reference for this representation theory is [FH].

The structure constants $c_{\lambda \mu}^{\nu}$ of this ring-with-basis, defined by

$$
\left[\mathrm{V}_{\lambda}\right]\left[\mathrm{V}_{\mu}\right]=\sum_{v} \mathrm{c}_{\lambda \mu}^{v}\left[\mathrm{~V}_{v}\right]
$$

are necessarily nonnegative (being the dimensions of certain vector spaces of intertwining operators), and there are many known rules for calculating them as the cardinalities of certain combinatorially defined sets. The most famous is the Littlewood-Richardson rule, which counts skew Young tableaux.

In several of these rules, the set being counted is the lattice points in a polytope (and in fact the polytopes from the different rules are linearly equivalent). The first was in the unpublished thesis $[\mathrm{J}]$, and was proved by establishing a bijection with skew Young tableaux; see also the appendix to [B]. It was rediscovered in [BZ1], where the proof starts with the (nonpositive) Steinberg rule for tensor products and uses an involution to cancel the negative terms. There is another extremely roundabout proof via the connection with Schubert calculus, for which a self-contained proof of a combinatorial rule was given in [KT2]. 
In this paper we give a new self-contained proof of this lattice-point-counting rule, in its incarnation as counting the hives from [KT1], whose definition we recall below. The main difficulty is in proving that the ring so defined (which is supposed to match up with $\left.\operatorname{Rep}\left(\mathrm{GL}_{n}(\mathbb{C})\right)\right)$ is associative. We give a bijective proof of this, using the octahedron rule from [RR, P, FZ, S]. This bijection was first found by CW in the honeycomb model, where the connection to the octahedron rule is not transparent.

Very recently, in $[\mathrm{S}]$, a closed form was found for compositions of the octahedron rule. In the last section we describe this formula in the special case relevant for this paper.

Since the octahedron rule is related to tropical algebraic geometry, we hope that our bijective proof of associativity will turn out to be the tropicalization of some natural but heretofore undiscovered birational map, as in [BZ3].

\subsection{Acknowledgements.}

It is our pleasure to thank Andrei Zelevinsky for comments on an earlier version of this paper, David Speyer for kindly working out the special case of his results $[\mathrm{S}]$ which appears in section 7, and Jim Propp for directing us to Speyer's preprint.

Note added in proof. Since acceptance of this paper, we learned of related results in $[\mathrm{NY}]$. We plan to explore this connection in a future paper.

\section{Hives}

Consider the triangle $\{[x, y, z]: x+y+z=n, x, y, z \geq 0\}$. This has $\left(\begin{array}{c}n+2 \\ 2\end{array}\right)$ integer points; call this finite set $\operatorname{tri}_{n}$. We will draw it in the plane and put $[n, 0,0]$ at the lower left, $[0, n, 0]$ at the top, and $[0,0, n]$ in the lower right. This triangle breaks up into $\left(\begin{array}{c}n+1 \\ 2\end{array}\right)$ right-side-up triangles $\overline{[x+1, y, z][x, y+1, z][x, y, z+1]}$ and $\left(\begin{array}{l}n \\ 2\end{array}\right)$ upside-down triangles $\overline{[x-1, y, z][x, y-1, z][x, y, z-1]}$. We will count certain integer labelings of tri $_{n}$ to compute Littlewood-Richardson coefficients, following [J], [BZ1], and especially [KT1].

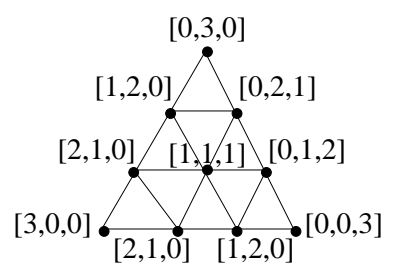

Figure 1: The set tri 3 , with its $\left(\begin{array}{c}3+1 \\ 2\end{array}\right)$ right-side-up and $\left(\begin{array}{l}3 \\ 2\end{array}\right)$ upside-down triangles.

A hive of size $n$ is a function $h: \operatorname{tri}_{n} \rightarrow \mathbb{Z}$ satisfying certain inequalities. Here are three equivalent ways to state those inequalities (of which we shall mainly use the first):

1. $h_{x+1, y, z+1}+h_{x, y+1, z+1} \geq h_{x+1, y+1, z}+h_{x, y, z+2}$ when these four points are all in $t r i_{n}$, and likewise for the $120^{\circ}$ and $240^{\circ}$ rotations of the hive. 
2. If you extend $h$ to a real-valued function on the solid triangle by making it linear on each little triangle $\overline{[x \pm 1, y, z][x, y \pm 1, z][x, y, z \pm 1]}, h$ is convex.

3. On each unit rhombus in the triangle, the sum across the short diagonal is greater than or equal to the sum across the long diagonal.

Note that the definition also makes sense for real-valued functions, in which case we will speak of a real hive. (We won't use this concept until section 5.)

Call these inequalities the rhombus inequalities on a hive. They naturally come in three families, according to the orientation of the rhombus.

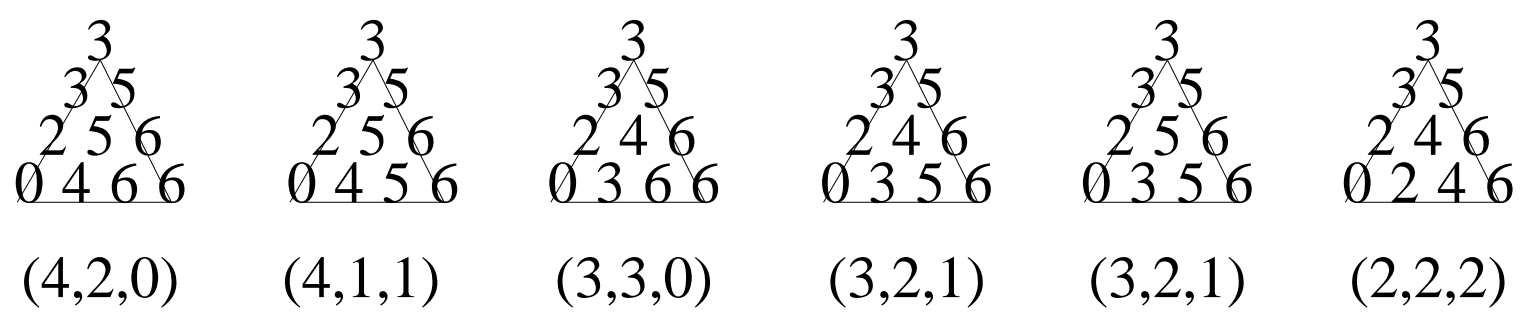

Figure 2: The hives with Northwest and Northeast side having differences $(2,1,0)$. The differences across the South side are indicated.

Definitions linearly equivalent to this one appeared first in [J, BZ1, BZ2]. This version from [KT1], like the one in [BZ2], has the benefit that each inequality only involves a constant number of entries (namely four), independent of $n$.

Proposition 1. Let $a_{0}, a_{1}, \ldots, a_{n}$ be the numbers on one side of a hive (read left-toright). Then $\mathrm{a}$ is convex, i.e. $\mathrm{a}_{\mathrm{i}} \geq \frac{1}{2}\left(\mathrm{a}_{\mathrm{i}-1}+\mathrm{a}_{i+1}\right)$. Put another way, the list $\left(\mathrm{a}_{1}-\right.$ $\left.a_{0}, a_{2}-a_{1}, \ldots, a_{n}-a_{n-1}\right)$ is a weakly decreasing list of integers.

Proof. There are two rhombi with an obtuse vertex at $a_{i}$. Adding the two corresponding rhombus inequalities, we get the desired result.

We can interpret such a list as a dominant weight for $\mathrm{GL}_{\mathfrak{n}}(\mathbb{C})$; call the set of such weights $\mathbb{Z}_{\text {dec }}^{n}$, and let $\lambda, \mu, v \in \mathbb{Z}_{\text {dec }}^{n}$ be three of them. Let $\operatorname{HIVE}_{\lambda \mu}^{v}$ denote the set of hives of size $\mathrm{n}$ such that

- the lower left entry is zero

- the differences on the Northwest side of the hive give $\lambda$

- the differences on the Northeast side of the hive give $\mu$

- the differences on the South side of the hive give $v$

where all differences are computed left-to-right throughout the paper. Note that for $\operatorname{HIVE}_{\lambda \mu}^{v}$ to be nonempty, we must have $\sum_{i}\left(\lambda_{i}+\mu_{i}\right)=\sum_{i} v_{i}$. The set $\bigcup_{v} \operatorname{HIVE}_{(2,1,0),(2,1,0)}^{v}$ is in figure 2 above.

Our goal is a self-contained proof of the following positive formula for $G L_{n}(\mathbb{C})$ tensor product multiplicities: 
Theorem 1. Let $\lambda, \mu, v \in \mathbb{Z}_{\text {dec }}^{n}$ and let $\mathrm{V}_{\lambda}, \mathrm{V}_{\mu}, \mathrm{V}_{v}$ be irreducible representations of $\mathrm{GL}_{\mathfrak{n}}(\mathbb{C})$ with those high weights. Then the number of times $\mathrm{V}_{v}$ appears as a constituent of the tensor product $\mathrm{V}_{\lambda} \otimes \mathrm{V}_{\mu}$ is the number of lattice points in $\mathrm{HIVE}_{\lambda \mu}^{v}$.

For example, figure 2 is computing the tensor square

$$
\mathrm{V}_{(2,1,0)}^{\otimes 2} \cong \mathrm{V}_{(4,2,0)} \oplus \mathrm{V}_{(4,1,1)} \oplus \mathrm{V}_{(3,3,0)} \oplus \mathrm{V}_{(3,2,1)}^{\oplus 2} \oplus \mathrm{V}_{(2,2,2)}
$$

While it doesn't make any sense to count real-valued hives with fixed boundary (which is why we insist on integer values), one can still consider the convex polytope thereof, and relate it to the geometry of certain moduli spaces (see the appendix to [KTW]). It is rather harder to formulate a "real version" of skew Young tableaux!

\section{$3 \quad$ Recognizing the representation ring $\operatorname{Rep}\left(\mathrm{GL}_{n}(\mathbb{C})\right)$}

Recall that the representation ring $\operatorname{Rep}\left(\mathrm{GL}_{n}(\mathbb{C})\right)$ has a basis $\left\{\left[\mathrm{V}_{\lambda}\right]\right\}, \lambda \in \mathbb{Z}_{\text {dec }}^{\mathrm{n}}$. Let $\omega_{i}^{n}$ denote the "fundamental weight" $(1, \ldots, 1,0, \ldots, 0)$ with $i 1$ s and $n-i 0$ s, the high weight of $\wedge^{i} \mathbb{C}^{n}$. (The notation is a little nonstandard - people usually just use $\omega_{i}$ - but that would be clumsy in lemma 2 to come.)

The only other facts we will need about $\operatorname{Rep}\left(\mathrm{GL}_{n}(\mathbb{C})\right)$ - for which our reference is $[\mathrm{FH}]$ - are that

- it is associative

- it is generated by the fundamental representations $\left[\mathrm{V}_{\omega_{i}^{\mathrm{n}}}\right]$ and $\left[\mathrm{V}_{(-1,-1, \ldots,-1)}\right]$

- $\left[\mathrm{V}_{\lambda}\right]\left[\wedge^{\mathfrak{n}} \mathbb{C}^{\mathfrak{n}}\right]^{-1}=\left[\mathrm{V}_{\lambda-(1, \ldots, 1)}\right]$ (we'll call this the $\operatorname{det}^{-1}$ rule)

- it satisfies the Pieri rule:

$$
\left[\mathrm{V}_{\lambda}\right]\left[\Lambda^{\mathfrak{i}} \mathbb{C}^{\mathfrak{n}}\right]=\bigoplus_{\substack{\pi \in\left\{0,11^{n}, \sum \pi=i \\ \lambda+\pi \in \mathbb{Z}^{n}\right.}} V_{\lambda+\pi}
$$

The sum is over those 0,1 -vectors $\pi$ with $i$ ones (or equivalently those weights occurring in $\left.\Lambda^{i} \mathbb{C}^{n}\right)$, such that $\lambda+\pi$ is weakly decreasing.

If $R$ is a ring-with-basis isomorphic to $\operatorname{Rep}\left(\mathrm{GL}_{\mathfrak{n}}(\mathbb{C})\right)$, then it satisfies the $\operatorname{det}^{-1}$ and Pieri rules; we now show that the converse is true. (Essentially the same observation is used in $[\mathrm{T}]$ and is surely much older.)

Proposition 2. Let $\mathrm{R}$ be a ring with $\mathbb{Z}$-basis $\left\{\mathrm{b}_{\lambda}\right\}, \lambda \in \mathbb{Z}_{\text {dec }}^{\mathfrak{n}}$, satisfying the $\operatorname{det}^{-1}$ and Pieri rules. Then the evident linear isomorphism $\phi: \operatorname{Rep}\left(\mathrm{GL}_{n}(\mathbb{C})\right) \rightarrow \mathrm{R},\left[\mathrm{V}_{\lambda}\right] \mapsto \mathrm{b}_{\lambda}$ is also a ring isomorphism. 
Proof. We want to show that $\phi(x y)=\phi(x) \phi(y)$. By linearity, it's enough to show it for $x$ a basis element $\left[\mathrm{V}_{\lambda}\right]$.

The Pieri and $\operatorname{det}^{-1}$ rules being true in both rings then tells us that this equation does hold if $x$ is a fundamental representation, $\left[\mathrm{V}_{(1, \ldots, 1,0, \ldots, 0)}\right]$ or $\left[\mathrm{V}_{(-1,-1, \ldots,-1)}\right]$.

More generally, let $y=b_{\mu_{1}} b_{\mu_{2}} \ldots b_{\mu_{l}}$ be a product of $l>0$ generators. Then

$$
\phi\left(b_{\lambda}\left(b_{\mu_{1}} b_{\mu_{2}} \ldots b_{\mu_{l}}\right)\right)=\phi\left(\left(b_{\lambda} b_{\mu_{1}} b_{\mu_{2}} \ldots b_{\mu_{l-1}}\right) b_{\mu_{l}}\right)=\phi\left(b_{\lambda} b_{\mu_{1}} b_{\mu_{2}} \ldots b_{\mu_{l-1}}\right) \phi\left(b_{\mu_{l}}\right)
$$

and induction on $l$ takes care of the rest. (Note that the identity, $\left[\mathrm{V}_{(0, \ldots, 0)}\right]$, is itself a product $\left[\mathrm{V}_{(1, \ldots, 1)}\right]\left[\mathrm{V}_{(-1, \ldots,-1)}\right]$ of two of our generators, so requiring $\mathrm{l}>0$ does not cause us to miss this basis element.)

So far we know that $\phi$ is establishing a ring isomorphism between the subspace of $\operatorname{Rep}\left(G_{n}(\mathbb{C})\right)$ generated by the fundamental representations, and the image of that under $\phi$. But since the fundamental representations generate $\operatorname{Rep}\left(G L_{n}(\mathbb{C})\right)$, and $\phi$ is a linear isomorphism, that's actually a ring isomorphism between the two rings.

In the rest of the paper our ring $R$ will be the hive ring, where the multiplication is defined by

$$
\mathrm{b}_{\lambda} \mathrm{b}_{\mu}=\sum_{\nu} \# \mathrm{HIVE}_{\lambda_{\mu}}^{v} \mathrm{~b}_{v}
$$

The hardest part in applying proposition 2 will be to prove that $R$ is associative. Since we haven't proved that yet it's a bit disingenuous to call it a ring, but we'll do it anyway rather than having to rename it afterward.

Once we've checked $\operatorname{det}^{-1}$, Pieri, and associativity for the hive ring, theorem 1 will follow from proposition 2 .

\section{The hive ring satisfies the $\operatorname{det}^{-1}$ and Pieri rules}

Lemma 1. Let $\mathrm{p}$ be a lattice parallelogram in the hive triangle $\mathrm{tri}_{\mathrm{n}}$, with edges parallel to the edges in the triangular lattice, and $\mathrm{h}$ a hive of size $\mathrm{n}$. Then the sum of $\mathrm{h}$ 's entries at the two obtuse angles of $\mathrm{p}$ is greater than or equal to the sum of $\mathrm{h}$ 's entries at the two acute angles of $\mathrm{p}$.

Proof. Add up all the rhombus inequalities from the rhombi inside and aligned with $\mathrm{p}$; everything cancels except the contributions from the four corners.

Proposition 3. In the hive ring, $\mathrm{b}_{\lambda} \mathrm{b}_{(-1, \ldots,-1)}=\mathrm{b}_{\lambda+(-1, \ldots,-1)}$. That is to say, the hive ring obeys the $\operatorname{det}^{-1}$ rule.

Proof. We're studying the hives with differences $\lambda_{i}=h_{n-i, 0, i}-h_{n-i+1,0, i-1}$ on the Northwest side, and that are linear with slope -1 on the Northeast side $\left(\right.$ so $\left.h_{0, n-z, z}=h_{0, n, 0}-z\right)$. We want to show there's exactly one, and it has $h_{i, 0, n-i}=h_{i, n-i, 0}-i$.

Let $h \in \operatorname{HIVE}_{\lambda,(-1, \ldots,-1)}^{\gamma}$ for some $v$. Consider the entry $h_{x, y, z}$, and the following two parallelograms in $\operatorname{tri}_{n}$ with $[x, y, z]$ as a vertex: 

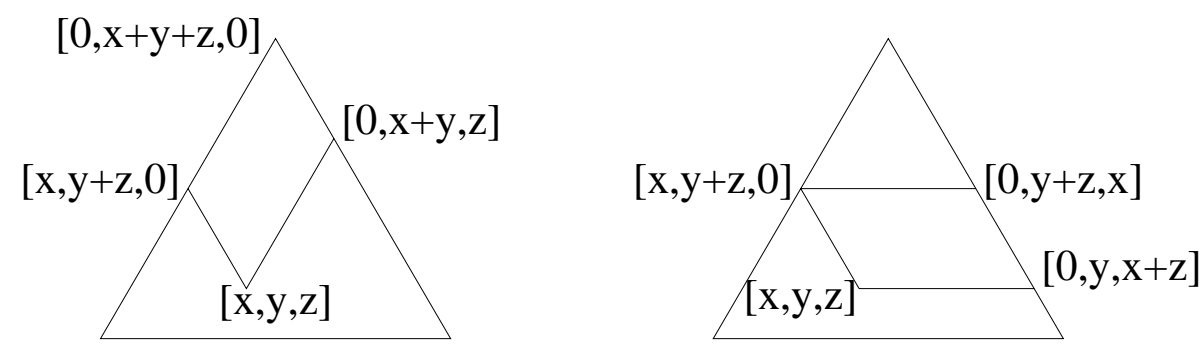

Let $\Lambda=\sum_{i} \lambda_{i}$ denote the value $h_{0, n, 0}$ at the top. Then the parallelogram inequalities of lemma 1 ,

$$
h_{x, y+z, 0}+h_{0, x+y, z} \geq h_{x, y, z}+h_{0, x+y+z, 0} \quad \text { and } \quad h_{x, y, z}+h_{0, y+z, x} \geq h_{x, y+z, 0}+h_{0, y, x+z}
$$

can be rewritten as

$$
h_{x, y+z, 0}+\Lambda-z \geq h_{x, y, z}+\Lambda \quad \text { and } \quad h_{x, y, z}+\Lambda-x \geq h_{x, y+z, 0}+\Lambda-x-z .
$$

These bound $h_{x, y, z}$ above and below by $h_{x, y+z, 0}-z$.

In particular the South edge is given by $h_{x, 0, z}=h_{x, z, 0}-z$; the only possible $h$ has the differences $\left(\lambda_{1}-1, \lambda_{2}-1, \ldots, \lambda_{n}-1\right)$ across the bottom and the rest of the hive is uniquely determined.

That shows uniqueness of the hive; how about existence? The convexity of the function $h_{x, y, z}=h_{x, y+z, 0}-z$ can be traced, with a bit of algebra, to the assumption that $\lambda$ was weakly decreasing.

This proposition can instead be proved by noting that adding an linear function of $y$ and $z$ to a hive produces a new hive, and by using the same inequalities to show that $b_{\lambda} b_{\overrightarrow{0}}=b_{\lambda}$, whose unique hive is constant on NW/SE lines.

Lemma 2. Let $\mathrm{h}$ be an $\mathrm{n}$-hive such that the differences down the NE edge are $\omega_{i}^{\mathrm{n}}$. Then the differences down the strip one step in from the $N E$ edge are either $\omega_{i}^{n-1}$ or $\omega_{i-1}^{n-1}$.

Depending on which, the last difference $\mathrm{h}_{1,0, \mathrm{n}-1}-\mathrm{h}_{0,0, \mathrm{n}}$ across the bottom either agrees with the last difference $\mathrm{h}_{1, n-1,0}-\mathrm{h}_{0, n, 0}$ on the $N W$ side, or is one larger, respectively.

Proof. For short, write $h_{1, n-1,0}, h_{0, n, 0}, h_{1,0, n-1}, h_{0,0, n}$ as $x, x+a, y, x+a+i$ respectively. (That $h_{0,0, n}=x+a+i$ follows from the assumption that the differences across the NE side are $\omega_{i}$, which has total $i$.)

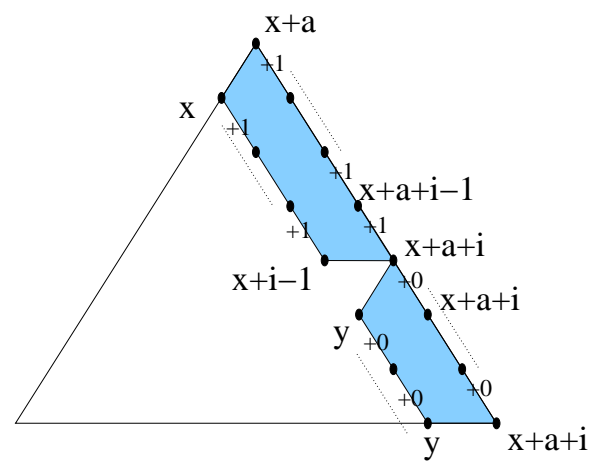


Using only the rhombus inequalities in the shaded regions of the figure above, and the same line of argument as in proposition 3 , we can show that $h_{1, n-1-i, i}=x+i$ and $h_{1, n-i-2, i+1}=y$. (These are the two adjacent interior entries indicated in the figure.)

Now the two rhombus inequalities relating those hive entries and the NE boundary say $x+i \geq y \geq x+i-1$. In particular, the difference $(x+a+i)-y$ is either $a$ or $a+1$, and that binary choice determines the rest of the strip.

Proposition 4. In the hive ring,

$$
b_{\lambda} b_{\omega_{i}}=\sum_{\substack{\pi \in\{0,1\}^{n}, \sum \pi=i \\ \lambda+\pi \in \mathbb{Z}_{\text {dec }}^{n}}} b_{\lambda+\pi} .
$$

In other words, "the hive ring obeys the Pieri rule".

Proof. Let $\mathrm{h}$ be a hive with differences $\lambda$ on the NW side, $\omega_{i}$ on the NE side. Rip off the NE strip from it and repeat, each time producing a hive one size smaller.

By inductive use of lemma 2, we see that the differences on the NE side go from $\omega_{i}$ (at size $n$ ) to $\omega_{0}$ (at size 0 ), so the differences across the bottom agree with $\lambda$ in $n-i$ places and are one larger in $i$ places. Moreover, the hive is uniquely determined by its labels on the bottom edge.

By proposition 1, the differences in the labels across the bottom are still decreasing. This, plus the previous paragraph, establishes the Pieri rule as an upper bound.

Given a 0,1 -string $\pi$ with $i$ ones such that $\lambda+\pi$ is dominant (and so should be giving a term in the Pieri rule), we can glue together the strips from lemma 2 and hope that we get a hive. The only rhombus inequalities left to check are those intersecting two adjacent strips, and we leave this to the reader.

\section{The hive ring is associative}

First off, what's the equation we're trying to prove? Let $h_{\lambda \mu}^{\sigma}=\# \operatorname{HIVE}_{\lambda \mu}^{\sigma}$, the structure constant in the hive ring. Then

$$
\left(b_{\lambda} b_{\mu}\right) b_{\nu}=\sum_{\sigma} h_{\lambda \mu}^{\sigma} b_{\sigma} b_{v}=\sum_{\sigma} \sum_{\pi} h_{\lambda \mu}^{\sigma} h_{\sigma \nu}^{\pi} b_{\pi}
$$

whereas

$$
b_{\lambda}\left(b_{\mu} b_{\nu}\right)=\sum_{\tau} b_{\lambda} h_{\mu \nu}^{\tau} b_{\tau}=\sum_{\tau} \sum_{\pi} h_{\mu \nu}^{\tau} h_{\lambda \tau}^{\pi} b_{\pi}
$$

Comparing coefficents of $b_{\pi}$, we see that we need to prove

$$
\sum_{\sigma} h_{\lambda \mu}^{\sigma} h_{\sigma \nu}^{\pi}=\sum_{\tau} h_{\mu \nu}^{\tau} h_{\lambda \tau}^{\pi}
$$

Consider a tetrahedron balanced perfectly on an edge, from directly above; the boundary of what you see is a square. Label the edges of this square (starting from the top left 
vertex and going clockwise) with the partial sums of $\lambda, \mu, v, \pi^{*}$. (The dominant weight $\pi^{*}$ is $\left(-\pi_{n},-\pi_{n-1}, \ldots,-\pi_{1}\right)$, the highest weight of the contragredient representation $\left(V_{\pi}\right)^{*}$. One could say it comes up because we're reading that edge of the hive backwards.)

If the top edge is labeled $\sigma$, then the number of ways of labeling the upper two faces with hives is $h_{\lambda \mu}^{\sigma} h_{\sigma v}^{\pi}$. Without fixing the labeling on that top edge, it's $\sum_{\sigma} h_{\lambda \mu}^{\sigma} h_{\sigma v}^{\pi}$. The corresponding statement for the lower two faces gives the other sum.
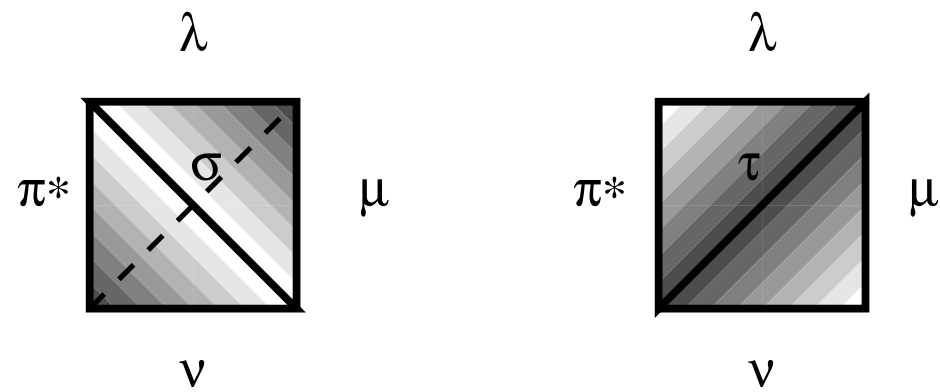

Theorem 2. There is a continuous, piecewise linear bijection between ways of labeling the upper two faces of this tetrahedron with a pair of real hives and ways of labeling the lower two faces, with given fixed labels $\lambda, \mu, \nu, \pi^{*}$ around the four non-horizontal edges.

Moreover, each formula for a label on a bottom face is a "tropical Laurent polynomial" in the entries on the top two faces, meaning it can be written as a maximum over some linear forms.

This bijection on matched pairs of real hives restricts to a bijection on matched pairs of integral hives, which establishes equation $(*)$ above.

Proof. This tetrahedron of size $\mathrm{n}$ breaks up into little tetrahedra, little upside-down tetrahedra, and octahedra (think about the $n=2$ case). In coordinates, let tet $_{\mathrm{n}}=$ $\left\{[x, y, z, w] \in \mathbb{N}^{4}: x+y+z+w=n\right\}$. Then the right-side-up tetrahedra have vertices

$$
[x+1, y, z, w],[x, y+1, z, w],[x, y, z+1, w],[x, y, z, w+1],
$$

the octahedra have vertices

$[x+1, y+1, z, w],[x+1, y, z+1, w],[x+1, y, z, w+1],[x, y+1, z+1, w],[x, y+1, z, w+1],[x, y, z+1, w+1]$ and the upside-down tetrahedra have vertices

$[x+1, y+1, z+1, w],[x+1, y, z+1, w+1],[x+1, y+1, z, w+1],[x+1, y+1, z+1, w]$.

Imagine the tetrahedron as initially being "full" of these pieces, which we will remove one by one from above, each being removable only when everything above is already out of the way. Along the way, we'll label all the interior lattice points with numbers. When we're done, leaving only the bottom two faces, it will turn out that we have two hives there.

Whenever we remove a little tetrahedron, we don't expose any new lattice points. Whenever we remove an octahedron, though, one of the old vertices (a local height max) 
goes with it and a new one becomes visible (a local height min). As we go, we label the vertices exposed according to the following formula:

$$
e^{\prime}:=\max (a+c, b+d)-e
$$

where $e$ was the label at the top, and $a, b, c, d$ the labels around the equatorial square. Our references for this octahedron rule are [P, FZ] (though it is much older, such as in $[\mathrm{RR}])$.

When we're done, we have labeled the bottom two faces. The process - which we call the excavation of tet $t_{n}$ - obviously provides its own inverse (the equation above is symmetric in $e$ and $e^{\prime}$ ), and preserves integrality.

It remains to see that what we get on the bottom is a pair of hives, i.e. satisfies the rhombus inequalities. We will show now that every unit rhombus in the tetrahedron gives a true rhombus inequality.

Say we've partially excavated, and every rhombus above the level so far dug out has satisfied this inequality. Now we extract a piece; this exposes some new rhombi that we need to check.

The $\mathrm{n}=2$ case. We remove the top two tetrahedra, then the octahedron, then a bottom tetrahedron. From the top, we see the labels
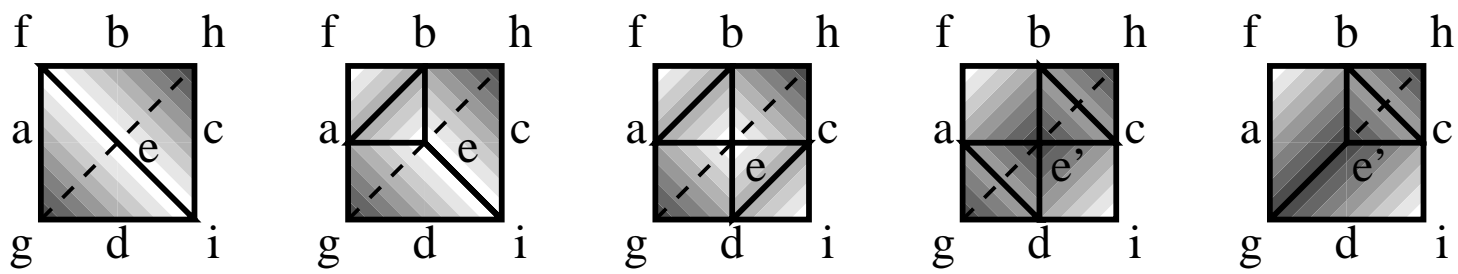

where the heavy (resp. dotted) lines indicate visible (resp. hidden) creases, and the shading indicates depth. From the South-Southeast ( $d$ in front, $b$ in back), the process looks like this:
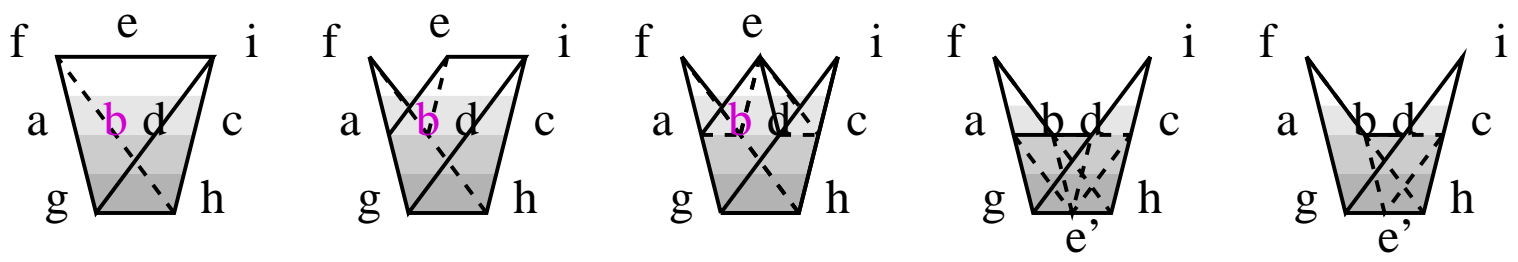

and at the end only the bce' $h$ tetrahedron is left.

The first two moves, removing the abef and edci tetrahedra, expose no new lattice points (only the creases change). The next move exposes the $e^{\prime}$ lattice point, and thus the rhombus with obtuse vertices $a, b$, acute $f, e^{\prime}=\max (a+c, b+d)-e$. We want to show that

$$
a+b \geq f+\max (a+c, b+d)-e
$$

or equivalently

$$
a+b \geq f+a+c-e, \quad a+b \geq f+b+d-e
$$

which follow from the $b+e \geq f+c, a+e \geq d+f$ inequalities on the top. 
The third move exposes the rhombus with obtuse vertices $a, e^{\prime}$, acute $b, g$. We want to show that

$$
a+\max (a+c, b+d)-e \geq b+g
$$

so it's enough to show one of them: $a+b+d-e \geq b+g$. This follows from $a+d \geq e+g$ on the top.

While we haven't explicitly handled all the rhombi in this size 2 tetrahedron - or even finished excavating it; the bhce' tetrahedron is still in place - the other rhombi are equivalent to these two under the evident $Z_{2} \times Z_{2}$ symmetries.

The general case. Any rhombus exposed fits into a size 2 tetrahedron, so we just have to apply the $\mathrm{n}=2$ case over and over.

Finally, we need the "tropical Laurent polynomial" statement. The rule $e^{\prime}=\max (a+$ $c, b+d)-e$ is the tropicalization of the subtraction-free rational function $E^{\prime}=(A C+$ $\mathrm{BD}) / \mathrm{E}$, meaning that,$+ \times, /$ have been replaced with $\max ,+,-$. As a very special case of the main theorem 1.6 in [FZ], one knows that if one uses this rational function recurrence during excavation, the labels on the bottom two faces are Laurent polynomials in the labels on the top two (rather than merely rational functions as one would expect).

Feeding this theorem and propositions 3 and 4 into proposition 2, we obtain theorem 1.

Since this paper was first written, it was proven in $[S]$ that the coefficients in these Laurent polynomials are all 1 , and the monomials identified. We state this result, in the special case relevant here, in section 7 .

\section{The honeycomb interpretation: scattering}

This section is distinctly less detailed than the others, and is largely for motivation. We recall briefly the honeycombs of [KT1], which are in $1: 1$ correspondence with hives, but better suited for some aspects of their study.

Let $\mathbb{R}_{\Sigma=0}^{3}$ denote the plane of triples of real numbers with zero sum. Define the coordinate directions in $\mathbb{R}_{\Sigma=0}^{3}$ to be parallel to $(0,1,-1)$, which we will draw as Northwest, $(-1,0,1)$, which we will draw as Northeast, and $(1,-1,0)$, which we will draw as South. A line segment oriented parallel to a coordinate direction has a constant coordinate, the one of the three coordinates constant along the edge.

A honeycomb is a measure on $\mathbb{R}_{\sum=0}^{3}$, constructed by summing the Lebesgue measure on a finite number of coordinate-oriented line segments (which may be unbounded), such that

- each unbounded ray goes in a coordinate direction (not its negative)

- around each point, the total "pull" of the up-to-six edges emanating from that point is the zero vector. 

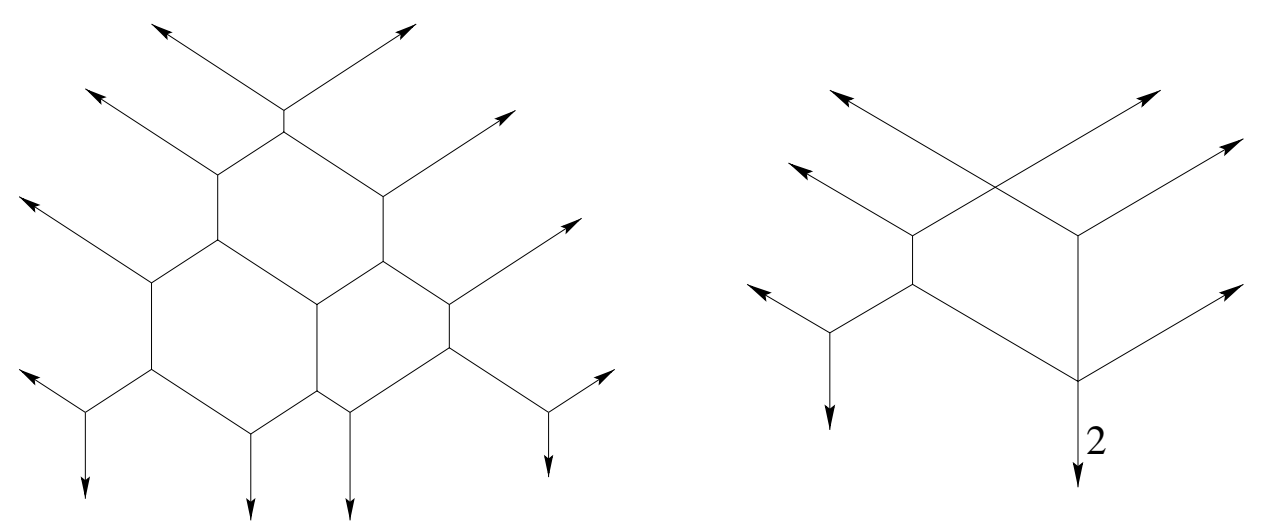

Figure 3: Two honeycombs, of sizes 4 and 3. The left one is more typical in having only $\mathrm{Y}$ vertices. All edges are multiplicity 1, except for the edge labeled 2 in the right-hand honeycomb.

Note that some of the segments may overlap (or even coincide), leading to multiplicities along the edges. Two honeycombs are displayed in figure 3.

Since any vertex of a honeycomb satisfies this "zero-tension" condition, the honeycomb as a whole does so (by a sort of Green's theorem), so the number of edges (counted with multiplicity) emanating in the Northwest, Northeast, or South directions must be the same number $n$. We call this the size of the honeycomb.

From a hive of size $\mathfrak{n}$, we construct a honeycomb of size $\mathrm{n}$ as follows. There is one honeycomb edge for each unit edge connecting two vertices in $\operatorname{tri}_{\mathfrak{n}}$, but perpendicular to it (living in the dual graph). The constant coordinate on that honeycomb edge is the difference of the two labels in the hive, up to a certain sign. To determine this sign, look for the unit triangle $\Delta$ in $\operatorname{tri}_{n}$ aligned with tri $i_{n}$, and containing the two hive labels and an extra vertex. The constant coordinate assigned is then the label on $\Delta$ counterclockwise of the extra vertex, minus the label on $\Delta$ clockwise of the extra vertex.

The vertices of the honeycomb then correspond to the linear regions in the hive. The rhombus inequalities on the hive, reinterpreted, state that the edges of the honeycomb are of nonnegative length. It is quite tricky to prove that this map from hives to honeycombs is in fact a bijection (theorem 1 of [KT1]).

We are now in a position to describe "honeycomb scattering", a honeycomb interpretation of the tetrahedron-evacuation bijection from section 5. This was the form in which one of us (CW) first found this proof of associativity.

Let HONEY ${ }_{\lambda \mu}^{v}$ denote the set of honeycombs whose boundary edges have constant coordinates $\lambda$ in the Northwest direction, $\mu$ in the Northeast direction, and $v$ in the South direction. To prove

$$
\sum_{\sigma} h_{\lambda \mu}^{\sigma} h_{\sigma \nu}^{\pi}=\sum_{\tau} h_{\mu \nu}^{\tau} h_{\lambda \tau}^{\pi}
$$


consider the set of pairs of honeycombs

$$
\left(h, h^{\prime}\right) \in \bigcup_{\sigma}\left(\operatorname{HONEY}_{\lambda \mu}^{\sigma} \times \operatorname{HONEY}_{\sigma \nu}^{\pi}\right) .
$$

We can draw such a pair $\left(h, h^{\prime}\right)$ by rotating $h^{\prime}$ by $180^{\circ}$, translating it some large distance in the $(1,-1,0)$ direction, and gluing it to $h$, as in the first entry in figure 4 . For reasons to be explained below we also compress this picture in the $y$ direction, making the edges all $90^{\circ}$ and $45^{\circ}$ from one another rather than $60^{\circ}$.

Now pull the lower honeycomb $h^{\prime}$ upwards, while sending the upper one downwards, at the same constant speed (this interpretation involves a time coordinate). At some point a vertex of $h^{\prime}$ will collide with one of $h$. We give an example of the whole process in figure 4, and (before they are given below) we invite the reader to guess the general rules defining scattering.

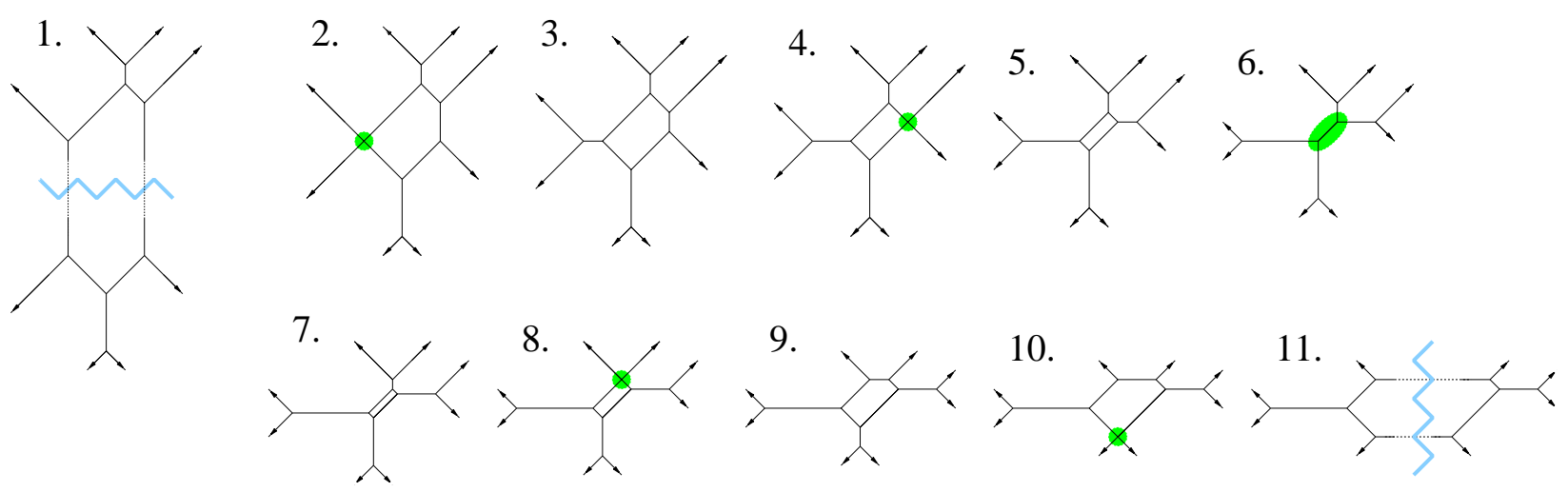

Figure 4: The eleven stages of two size 2 honeycombs scattering off one another. The collisions are circled. Before the double collision in (6), the rectangle is shrinking; after that collision, the rectangle bounces back out.

The rules for scattering are as follows. Each vertex in $h$ (respectively, $h^{\prime}$ ) is given an initial velocity of down (respectively, up). All vertices move until there is a collision.

Generically, the first sort of collision met is that of a single edge contracting (as in (1-2), (3-4), (7-8), (10-11) of figure 4). When this happens, we redirect the vertically colliding particles to move left and right, conserving total energy and momentum. Note that what used to be a vertical line connecting them is now horizontal.

After some of these collisions, a second type of collision is possible, involving two pairs of converging vertices making a rectangle collapse (as in (5-6) of figure 4). These are again redirected out, conserving energy and momentum.

(For generic $h$ and $h^{\prime}$, these two are the only sorts of collisions that can happen during the whole scattering. Nongeneric $h, h^{\prime}$ can be understood by taking limits from the generic case, so we won't dwell on them.)

It is true at the beginning, and remains true after either type of collision, that if a vertex is attached to a vertical line then it is moving vertically and will eventually get into a 2-vertex collision. Note that each 2-vertex collision increases the number of particles 
moving horizontally and decreases the number of vertical edges, and each 4 -vertex collision decreases the number of particles moving directly toward one another.

So there are only a finite number of collisions; when the scattering is over, all particles are moving horizontally, there are no vertical edges, and all the left-moving particles are left of all the right-moving particles. At this point we can cut the diagram in half along the growing edges, and we get two new squashed honeycombs - except that they've been squashed along the $x$ direction rather than the $y$.

\subsection{Honeycomb scattering vs. hive excavation.}

These two types of collisions - 2-vertex and 4-vertex - correspond to the tetrahedral and octahedral excavations in theorem 2 . The hive labels are linearly related to the constant coordinates on the diagonal lines. Note that during a 2-vertex collision, the four diagonal lines incident move at a constant speed - on the excavation side, this reflects the fact that excavating a tetrahedron exposes no new vertices and requires no new labeling. The max of the octahedron rule is implemented by the two ways a rectangle can collapse whichever one comes first determines how the vertices bounce back out.

In the hive excavation picture, it is easier to deal with degenerate cases uniformly the octahedron rule still applies, it just happens that the max involved is achieved twice. Also the hive picture doesn't introduce this spurious "time" coordinate; in particular, the excavation of the large tetrahedron can be done in many different ways, all giving the same answer.

On the other hand, in the honeycomb picture it is more manifest that the limiting object after scattering is again two honeycombs glued together, rather than having to check the rhombus inequalities in various cases, as occurred in theorem 2.

\subsection{The scattering rule in $[\mathrm{GP}]$.}

A very similar result is proven in $[\mathrm{GP}]$, though phrased in terms of braid relations rather than excavation. It is less immediately obvious that their rule is constructing an associator, since it involves six inputs rather than four. In fact their pseudo-line arrangements can be corresponded to a partially excavated cube of size $n$, rather than our tetrahedron, and their braid move corresponds to the removal of one little cube.

\section{A closed form for the associativity bijection}

As promised in section 5, we give a closed-form expression for each entry of the bottom two hives as a "tropical Laurent polynomial" (a single maximum over a family of linear expressions) in the entries of the top two.

This is a special case of a general result proved by Speyer in [S], conjectured in [FZ]: during any order in which we excavate a tetrahedron, a label $b$ exposed at time $t_{2}>t_{1}$ is a Laurent polynomial with positive coefficients in those labels on the surface at time $t_{1}$. Speyer proves a more precise conjecture due to Propp: each Laurent monomial in $b$ 
corresponds to a matching of a certain graph $\mathrm{G}_{\mathrm{b}}$, meaning a subset of the edges covering each vertex exactly once, where the graph $G_{b}$ is determined by the $t_{1}$-surface and the $t_{2}$-entry. Intriguingly, the "graphs with open faces" $G_{b}$ that Speyer constructs to prove this look like partially-scattered honeycombs!

Consider the graph G (with some unbounded edges) constructed by scattering two standard $\mathfrak{n}$-honeycombs (meaning, all finite edges of length 1 ) off one another, stopping exactly when the first $n$ collisions occur (simultaneously). This graph has $n-1$ rhombi and two triangular arrays of $\left(\begin{array}{c}n-1 \\ 2\end{array}\right)$ hexagons. The $\mathfrak{n}=4$ example is drawn in figure 5 . Its regions correspond to the labels on the top two faces of a tetrahedron to be excavated.
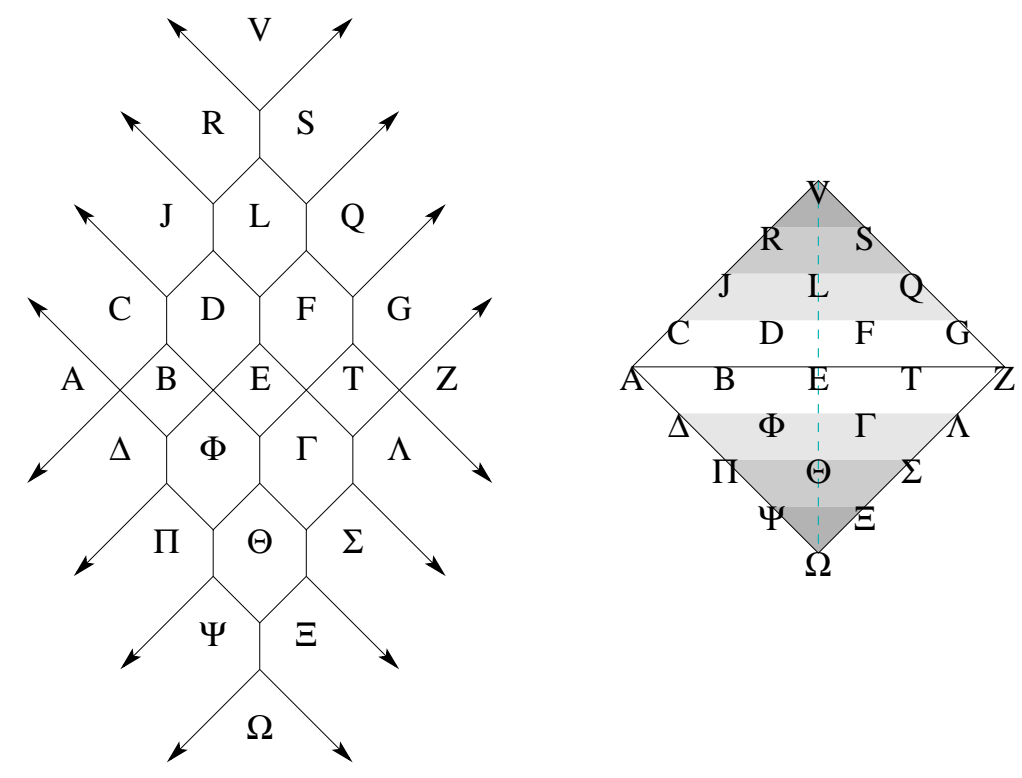

Figure 5: Two standard 4-honeycombs caught at the moment of first scattering (left). The regions correspond to labels on the top two faces of a tetrahedron (right), as indicated.

To expose a bottom entry $b$, there is a unique minimal set of unit tetrahedra and octahedra that must be excavated. For example, to expose the bottom entry below the $\mathrm{F}$ in figure 5, one must remove the octahedra with top points labeled by T, E, F.

We are now ready to describe the graph $G_{b}$ that Speyer associates to a bottom entry $\mathrm{b}$ : it is the minimal subgraph of $\mathrm{G}$ enclosing those entries that must be excavated to get to $b$. In fact one must also consider the adjoining hexagonal regions in Speyer's definition of $\mathrm{G}_{\mathrm{b}}$ as a "graph with open faces". See figure 6 for the case $\mathrm{b}$ being the entry below the F.

Given such a graph $\mathrm{G}_{\mathrm{b}}$, and a matching $\mu$ of it, Speyer defines the matching (Laurent) monomial $m_{\mu}$ as the product over all faces of $G_{b}$ (including the exterior hexagons), of the corresponding variable raised to the power

- one minus the number of adjacent edges in $\mu$, for a rhombus or external hexagon, or

- two minus the number of adjacent edges in $\mu$, for an interior hexagon. 


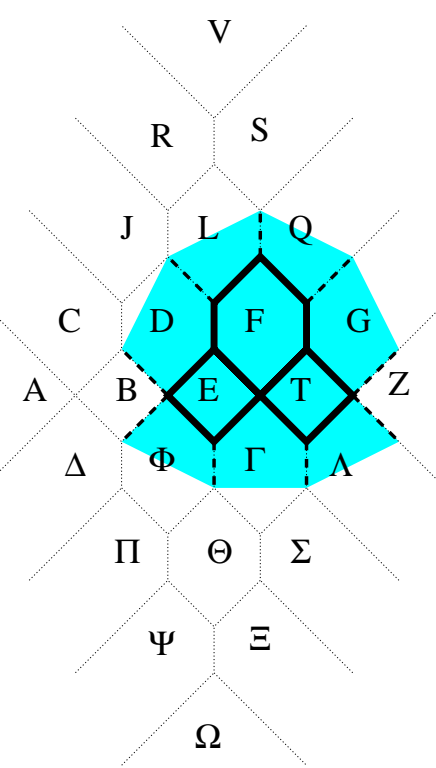

Figure 6: The graph with open faces whose matchings will calculate the entry directly below the label $\mathrm{F}$, with three interior and seven exterior faces.

In figure 7 we draw all the matchings of our $\mathrm{G}_{\mathrm{b}}$ from figure 6 , and compute the matching monomials.

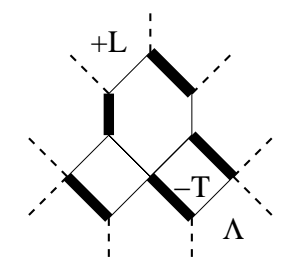

$\frac{\mathrm{L} \Lambda}{\mathrm{T}}$

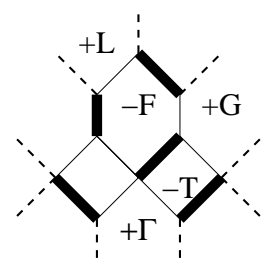

$\frac{\mathrm{GL} \Gamma}{\mathrm{TF}}$

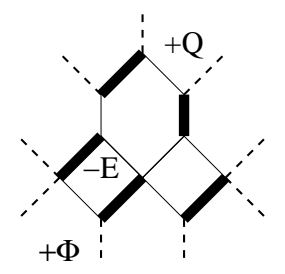

$\frac{\mathrm{Q} \Phi}{\mathrm{E}}$

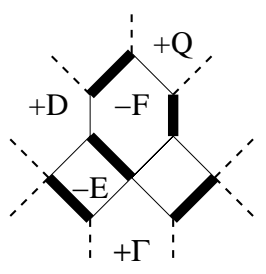

$\frac{\mathrm{QD} \Gamma}{\mathrm{EF}}$

Figure 7: The matchings of $\mathrm{G}_{\mathrm{b}}$ - in each figure, each vertex touches exactly one heavy edge - and their matching monomials.

Speyer's theorem (for our case) now reads as follows:

Theorem 1. [S] Let $\mathrm{b}$ be a label on the bottom of the hive tetrahedron, and $\mathrm{G}_{\mathrm{b}}$ the minimal subgraph of $\mathrm{G}$ enclosing the entries on the top that must be excavated to expose $\mathrm{b}$.

Then if during excavation, we use the rational function octahedron recurrence $\mathrm{E}^{\prime}=$ $\mathrm{E}^{-1}(\mathrm{AC}+\mathrm{BD})$, the resulting value of $\mathrm{b}$ can be computed as a sum over all matchings $\{\mathrm{m}\}$ of $\mathrm{G}_{\mathrm{b}}$, of the associated matching monomials $\left\{\mu_{\mathrm{m}}\right\}$.

If we instead use the tropical recurrence, the value of $\mathrm{b}$ can be computed as a maximum over all matchings, of the corresponding linear forms. 
To compute the figure 6 example directly: the octahedron recurrence gives

$$
\begin{aligned}
\mathrm{T}^{\prime} & =\mathrm{T}^{-1}(\mathrm{~F} \wedge+\mathrm{G} \Gamma) \\
\mathrm{E}^{\prime} & =\mathrm{E}^{-1}(\mathrm{~F} \Phi+\mathrm{D} \Gamma) \\
\mathrm{F}^{\prime} & =\mathrm{F}^{-1}\left(\mathrm{~T}^{\prime} \mathrm{L}+\mathrm{QE} \mathrm{E}^{\prime}\right) \\
& =\mathrm{F}^{-1}\left(\mathrm{~T}^{-1}(\mathrm{~F} \wedge+\mathrm{G} \Gamma) \mathrm{L}+\mathrm{QE}^{-1}(\mathrm{~F} \Phi+\mathrm{D} \Gamma)\right) \\
& =\mathrm{T}^{-1} \wedge \mathrm{L}+\mathrm{F}^{-1} \mathrm{~T}^{-1} \mathrm{G} \Gamma \mathrm{L}+\mathrm{QE}^{-1} \Phi+\mathrm{F}^{-1} \mathrm{QE}^{-1} \mathrm{D} \Gamma
\end{aligned}
$$

which agrees with the theorem, being the sum of the terms from figure 7 . The tropical version is therefore

$$
\mathrm{F}^{\prime}=\max \{-\mathrm{T}+\Lambda+\mathrm{L},-\mathrm{F}-\mathrm{T}+\mathrm{G}+\Gamma+\mathrm{L}, \mathrm{Q}-\mathrm{E}+\Phi,-\mathrm{F}+\mathrm{Q}-\mathrm{E}+\mathrm{D}+\Gamma) .
$$

\section{References}

[BZ1] A. Berenstein, A. Zelevinsky, Involutions on Gel'fand-Tsetlin schemes and multiplicities in skew $\mathrm{GL}_{\mathrm{n}}$-modules. (Russian) Dokl. Akad. Nauk SSSR 300 (1988), no. 6, 1291-1294; translation in Soviet Math. Dokl. 37 (1988), no. 3, 799-802.

[BZ2] A. Berenstein, A. Zelevinsky, Triple multiplicities for $\mathrm{sl}(\mathrm{r}+1)$ and the spectrum of the exterior algebra of the adjoint representation. J. Algebraic Combin. 1 (1992), no. $1,7-22$.

[BZ3] A. Berenstein, A. Zelevinsky, Tensor product multiplicities, canonical bases and totally positive varieties, Invent. Math., 143 (2001), no. 1, 77-128.

[B] A. Buch, The saturation conjecture (after A. Knutson and T. Tao). With an appendix by William Fulton. Enseign. Math. (2) 46 (2000), no. 1-2, 43-60.

[FZ] S. Fomin, A. Zelevinsky, The Laurent phenomenon. Adv. in Appl. Math. 28 (2002), no. $2,119-144$.

[FH] W. Fulton, J. Harris, Representation theory, Springer-Verlag (1991).

[GP] O. Gleizer, A. Postnikov, Littlewood-Richardson coefficients via Yang-Baxter equation, International Mathematics Research Notices (2000), no. 14, 741-774.

[J] S. Johnson, The Schubert calculus and eigenvalue inequalities for sums of Hermitian matrices, Ph. D. thesis, U. C. Santa Barbara, 1979.

[KT1] A. Knutson, T. Tao, The honeycomb model of $\mathrm{GL}_{n}(\mathbb{C})$ tensor products I: proof of the saturation conjecture, J. of Amer. Math. Soc, 12 (1999), no. 4, 1055-1090.

[KT2] A. Knutson, T. Tao, Puzzles and (equivariant) cohomology of Grassmannians, Duke Math. J. 119 (2003), no. 2, 221-260. 
$[\mathrm{KTW}]$ A. Knutson, T. Tao, C. Woodward, The honeycomb model of $\mathrm{GL}_{n}(\mathbb{C})$ tensor products II: facets of the L-R cone, J. Amer. Math. Soc. 17 (2004), no. 1, 19-48.

[NY] M.Noumi, Y.Yamada, Tropical Robinson-Schensted-Knuth correspondence and birational Weyl group actions, Adv. Stud. in Pure Math. 40 (2004), 371-442.

[P] J. Propp, The many faces of alternating-sign matrices, in Discrete Models: Combinatorics, Computation, and Geometry, Discrete Math. Theor. Comput. Sci. Proc., AA, Maison Inform. Math. Discrét. (Paris, 2001), 43-58,

[RR] D. P. Robbins, H. Rumsey, Determinants and alternating-sign matrices, Advances in Math. 62 (1986) 169-184.

[S] D. Speyer, Perfect matchings and the octahedron recurrence, preprint May 2003.

[T] H. Tamvakis, The connection between representation theory and Schubert calculus, preprint 2002. 